\title{
Pneumomediastinum after functional endoscopic sinus surgery under general anesthesia -A case report-
}

\author{
Jung Man Park, Young-Chul Park, Jong Nam Lee, Jun Seok Bae, and Shin Kyu Kang \\ Department of Anesthesiology and Pain Medicine, St. Mary's Medical Center, Busan, Korea
}

The occurrences of pneumothorax and pneumomediastinum are rare, but considered to be potentially lifethreatening conditions in patients undergoing functional endoscopic sinus surgery under general anesthesia. Tracheobronchial rupture may results in serious complications, such as pneumothorax and pneumomediastinum. It may occur accidentally by endotracheal tube when the patient's neck is flexed or extended. We report the case of a 48-year-old female patient who developed massive subcutaneous emphysema, pneumothorax, pneumomediastinum and pneumoperitoneum seven hours after functional endoscopic sinus surgery under general anesthesia. (Korean J Anesthesiol 2013; 64: 367-372)

Key Words: Pneumomediastinum, Pneumothorax, Tracheobronchial rupture, Valsalva maneuver.

Endotracheal intubation is the most basic and important procedure for anesthesiologists in emergency situations as well as for routine situations in the operating room and intensive care unit. Generally, the location of the tube after endotracheal intubation is verified using physical methods by checking the gradations on the tube, auscultation of both lungs, and observing symmetric expansion of the chest during respiration, and various indicators such as ECG, oxygen saturation, airway pressure, positive end expiratory pressure, and end-tidal carbon dioxide tension. However, even when the tube is properly positioned, a change in the position of the head or neck can cause accidental extubation or deep intubation, leading to a serious situation for the patient. Various monitoring equipment monitors the patient during surgery so when the location of the tube is altered, it is quickly noticed. However, airway damage caused by tube movement during extubation only appears as clinical symptoms after a certain amount of time has lapsed; hence, there is need for more caution. There are many domestic and foreign cases which have reported complications caused by deep intubation, but it is rare to find cases where airway damage occurred when the endotracheal tube moved into the bronchus due to bucking while regaining consciousness. The authors experienced a case where functional endoscopic sinus surgery and septoplasty were performed under general anesthesia for sinusitis and nasal septal deviation, and complications including subcutaneous emphysema, pneumothorax, pneumo-

Received: April 2, 2012. Revised: 1st, May 15, 2012; 2nd, June 8, 2012. Accepted: June 21, 2012.

Corresponding author: Young-Chul Park, M.D., Department of Anesthesiology and Pain Medicine, St. Mary's Medical Center, Yongho-dong, Nam-gu, Busan 608-090, Korea. Tel: 82-51-933-7363, Fax: 82-51-932-8600, E-mail: pyc9@yahoo.co.kr

C) This is an open-access article distributed under the terms of the Creative Commons Attribution Non-Commercial License (http:// creativecommons.org/licenses/by-nc/3.0/), which permits unrestricted non-commercial use, distribution, and reproduction in any medium, provided the original work is properly cited. 
mediastinum, and pneumoperitoneum occurred 7 hours postoperatively; hence, we are reporting the case together with a review of the medical literature.

\section{Case Report}

A 48-year-old female patient $158 \mathrm{~cm}$ in weight weighing $53 \mathrm{~kg}$ was admitted to receive functional endoscopic sinus surgery and septoplasty for a diagnosis of chronic sinusitis and nasal septal deviation. The patient was a non-smoker and her medical history stated that she had received an appendectomy one year prior to her visit and had a benign tumor removed from her bladder six months before her visit. There were no abnormalities other than light anemia with an $\mathrm{Hb}$ of $9.7 \mathrm{~g} / \mathrm{dl}$ and Hct at $30.7 \%$ from the blood test.

Glycopyrrolate $0.2 \mathrm{mg}$ was IM injected as premedication, and preoxygenation was sufficiently achieved with $100 \%$ oxygen before induction of anesthesia. Propofol $100 \mathrm{mg}$ and rocuronium $50 \mathrm{mg}$ was slowly IV injected and after fully confirming loss of consciousness and muscle relaxation, oral endotracheal intubation was attempted with a curved laryngoscope (Macintosh size3 blade). The patient was class 1 based on the Cormack and Lehane laryngeal endoscopic classification, and endotracheal intubation was performed smoothly in the first attempt with a preformed Ring, Adair, Elwyn tube (RAE, Mallinckrodt) $7.0 \mathrm{~mm}$ in diameter. Proper placement of the tube was confirmed by chest auscultation and it was fixed at a tube length of $20 \mathrm{~cm}$. A tidal volume of 450 $\mathrm{ml}$ and respiration rate of 12 times/min were maintained with mechanical ventilation (Aestiva $/ 5^{\circledR}$, Datex-Ohmeda, Madison, USA); airway pressure was $14-16 \mathrm{cmH}_{2} \mathrm{O}$ and positive end expiratory pressure was $1 \mathrm{cmH}_{2} \mathrm{O}$. Anesthesia was maintained with $\mathrm{O}_{2} 2 \mathrm{~L} / \mathrm{min}, \mathrm{N}_{2} \mathrm{O} 2 \mathrm{~L} / \mathrm{min}$, and sevoflurane $2.0-2.5$ vol\%. During surgery, the blood pressure was 105-150/60-95 $\mathrm{mmHg}$, heart rate $75-100$ beats/min, and oxygen saturation 99-100\%, and end-tidal carbon dioxide tension was maintained at $33-37 \mathrm{mmHg}$. The duration of the surgery was 2 hours and 30 minutes, and there were no abnormalities during surgery. After surgery, pyridostigmine $15.0 \mathrm{mg}$ and glycopyrrolate 0.4 mg were IV injected to reverse the muscle relaxation. When spontaneous respiration was sufficiently recovered and extubation of the endotracheal tube was about to be performed, suddenly, the patient severely shook her head and started to struggle so the bandage fixing the tube was immediately removed; air was removed from the balloon, and extubation was performed. During extubation, the patient was severely bucking while raising her neck and upper body so surrounding staff were called for help in safely holding down the patient. After extubation, the patient was soon stabilized after a few coughs and was moved to the recovery room. A blood pressure of $120-140 / 70-80 \mathrm{mmHg}$, heart rate of $65-76$ beats/min, and oxygen saturation of $99-100 \%$ were maintained in the recovery room, and other than weak pain in the operated area, there were no specific complaints of symptoms so she was moved to general ward without abnormalities. Three hours after surgery, the patient started to feel nauseous and five hours after surgery, she vomited severely several times thus metoclopramide 10 mg was IV injected. Seven hours after surgery, the patient's neck and face swelled up with complaints of pain, and physical examination showed crepitation. Vital signs at the time was blood pressure $100 / 60 \mathrm{mmHg}$, heart rate 80 beats $/ \mathrm{min}$, respiration rate 20 times $/ \mathrm{min}$, temperature $36.3^{\circ} \mathrm{C}$, and oxygen saturation $98 \%$. Arterial blood gas analysis was immediately performed as well as chest and neck x-ray, and neck CT. The
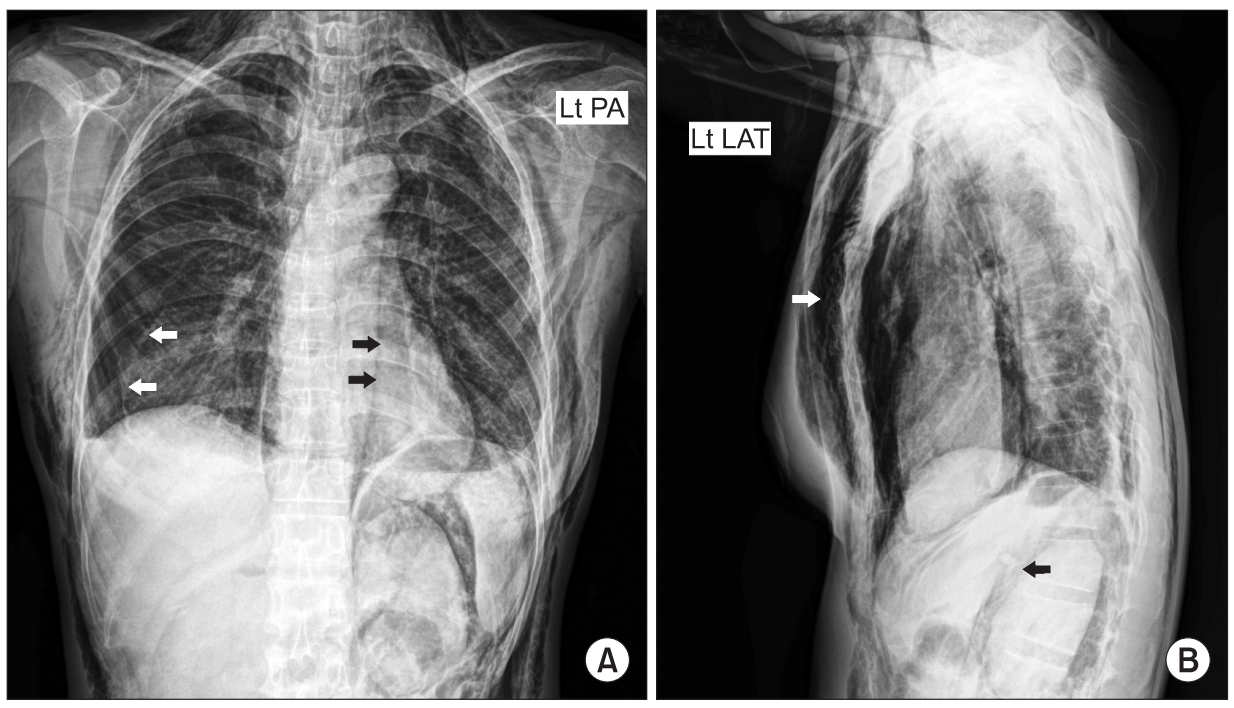

Fig. 1. The postoperative chest PA $\mathrm{X}$-ray (A) shows massive subcutaneous emphysema, pneumothorax (white arrows) and lucent band of gas that extends along descending aorta (black arrows). Left lateral view of chest X-ray (B) shows subcutaneous emphysema (white arrow) and pneumoperitoneum (black arrow). 


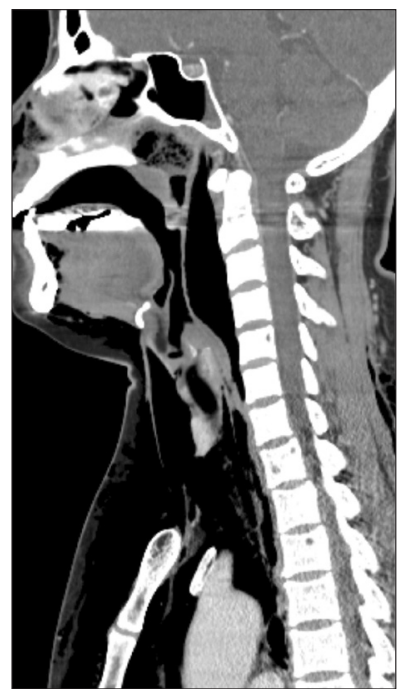

Fig. 2. Neck CT shows retropharyngeal air and extensive subcutaneous emphysema in neck.

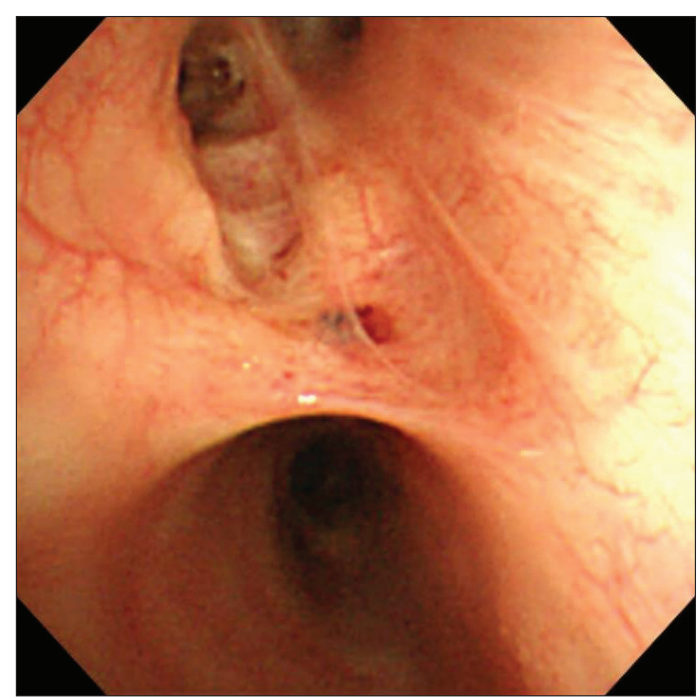

Fig. 3. Fiberoptic bronchoscopy shows round shape mucosal defect and bloody discharge in right second carina.

patient was put in semi-Fowler's position to provide oxygen $2 \mathrm{~L} /$ min through a nasal cannula, and was put on a fast. Results of arterial blood gas analysis showed $\mathrm{pH} 7.42, \mathrm{PaCO}_{2} 31.7 \mathrm{mmHg}$, $\mathrm{PaO}_{2} 80.2 \mathrm{mmHg}, \mathrm{HCO}_{3}{ }^{-} 20.7 \mathrm{mEq} / \mathrm{L}$, and $\mathrm{SaO}_{2} 96.8 \%$. In the chest x-ray pneumothorax, subcutaneous emphysema in the chest wall, pneumomediastinum, and pneumoperitoneum were observed (Fig. 1). The neck CT showed extensive subcutaneous emphysema in the neck and face area, and emphysema in the retropharyngeal space (Fig. 2). The patient was immediately transferred to ICU and oxygen $5 \mathrm{~L} / \mathrm{min}$ was provided through a mask in Fowler's position. No abnormalities were discovered in the fiberoptic laryngoscope examination

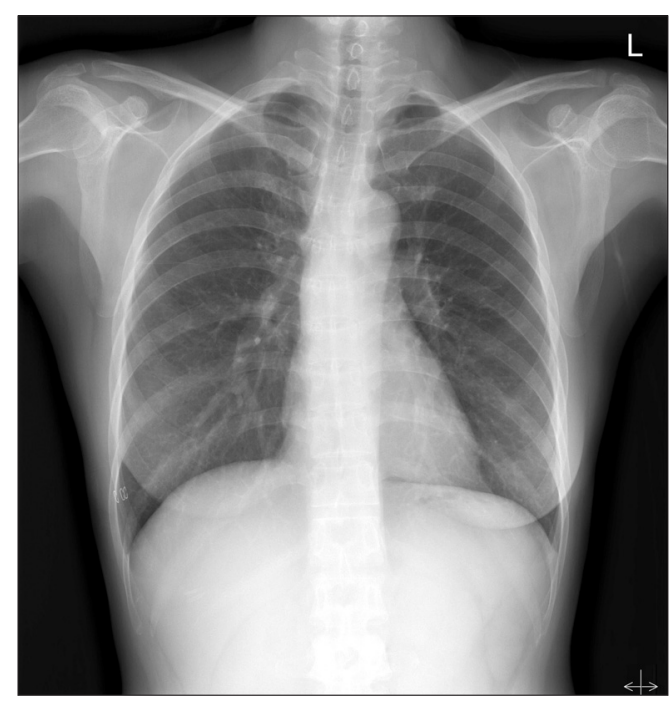

Fig. 4. Follow up chest PA X-ray shows resolution of the pneumomediastinum two weeks later.

performed by the ENT department when investigating the cause. $4 \mathrm{ml}$ of air were extracted from the retropharyngeal space with an $18 \mathrm{G}$ needle using the intraoral approach, and the thoracic surgery performed insertion of chest tube on the right chest and incision of the subcutaneous emphysema on the chest wall. The patient received esophago gastro duodenoscopy (EGD) and bronchoscopy for differential diagnosis, and there were no abnormalities found in the EGD. In the bronchoscopy, there were no abnormalities in the laryngopharynx, vocal cords, and carina, but mucosal damage and bloody secretion were observed in the right second carina (Fig. 3). In the bronchial sputum examination, the patient was positive for mycobacterium tuberculosis polymerase chain reaction, and was diagnosed with tuberculosis. The patient received conservative treatment and anti-tubercular treatment, and was discharged from the hospital 2 weeks later with improved symptoms (Fig. 4).

\section{Discussion}

Clinical symptoms of pneumomediastinum are usually chest pain with accompanying radiating pain from the neck or back, breathing difficulties, tachypnea, and throat discomfort. Physical findings include subcutaneous emphysema, the loss of cardiac dullness to percussion, and mediastinal crepitation (Hamman's sign) [1,2]. Diagnosis can be achieved by the above mentioned clinical symptoms and radiological observations. In a simple chest x-ray, usually a line due to an air shadow can appear along the left heart disinsertion and between the heart and diaphragm (continuous diaphragm sign), while in the lateral view of chest x-ray, there can be subcutaneous emphysema of the chest and neck, air shadows 
that appear along the back of the sternum or aortic arches, and air shadow around the outer pericardium of the right pulmonary artery (ring around the artery sign) [3]. Fifty percent of pneumomediastinum cannot be found just with chest PA $\mathrm{X}$-rays; therefore, a lateral chest X-ray must be taken together [1]. Chest CT can clarify the diagnosis or help confirm lesions that did not appear in simple chest x-rays [3].

Zylak et al. [3] explain the occurrence of pneumomediastinum by the elements inside the thorax and by the elements outside the thorax, depending on the place where the air originated. Elements inside the thorax are rupturing the pulmonary alveoli from asthma, vomiting, giving birth, lifting heavy objects, and applying force with the glottis closed, as well as secondary rupture of pulmonary alveoli or damage to the trachea and bronchus from external physical impact. Elements outside the thorax are cases where anatomical boundaries surrounding the mediastinum is pierced and air is injected, such as in surgery or injury to the head and neck area, tooth extraction, infection or penetration in the abdominal or peritoneal cavity. From these various causes, those that are associated with surgery and anesthesia can be complications from the surgery itself, pressure damage from positive ventilation during anesthesia, airway damage from endotracheal intubation, and airway damage from movement of an endotracheal tube.

One case of pneumomediastinum that occurred after functional endoscopic sinus surgery was reported by Sohail et al. [4], where a few hours after receiving functional endoscopic sinus surgery under general anesthesia, pneumomediastinum occurred together with subcutaneous emphysema in the face, neck, and chest wall, and the patient recovered after conservative treatment. The authors inferred that the surgery itself could not be seen as the direct cause of pneumomediastinum, and that pulmonary alveoli rupture or airway damage from endotracheal intubation were possible causes. Also in our case, the fiberoptic laryngoscope examination performed by the ENT department showed no abnormalities from the operated area to the glottis, so there is low possibility that the surgery itself was the direct cause for pneumomediastinum.

The possibility of pressure damage from positive ventilation during anesthesia is also low since various indicators such as ECG, oxygen saturation, airway pressure, positive end expiratory pressure, and end-tidal carbon dioxide tension were all in the normal range.

The possibility of mucosal damage due to endotracheal intubation while inducing anesthesia is also low since there were no difficulties in the intubation process and the $7.0 \mathrm{~mm}$ diameter RAE tube forms a curve at a $20 \mathrm{~cm}$ length so it is difficult to have deeper intubation. In addition, in the research results of Song et al. [5], when mean height of Korean females is $157.8 \pm 3.7$ $\mathrm{cm}$; the mean length from the front teeth to carina was $25.3 \pm 0.9$ $\mathrm{cm}$, and the height of the patient in our case was $158 \mathrm{~cm}$ so the possibility of deep intubation is low. Additionally, if the tube had been inserted into right second carina, there should have been a clear reduction in breath sound in the non-ventilated lung, increased airway pressure, and a decrease in oxygen saturation, but these situations did not occur up to the end of surgery. Therefore, the authors believe that there is low possibility of mucosal damage due to endotracheal intubation. In addition, since a stilet was not used, damage from a stilet can also be ruled out.

Next, there is the possibility of mucosal damage due to the movement of the endotracheal tube. In a study regarding the movement of the endotracheal tube, Conrardy et al. [6] reported that the tube moves $1.5 \mathrm{~cm}$ towards the carina when bending the neck, and moves $2.4 \mathrm{~cm}$ towards the vocal cords when the neck is extended. In our case, there were no excessive position changes to the neck area during surgery, but in the process of regaining consciousness and spontaneous respiration, there were several bouts of severe struggling and coughing before extubation. The tube could have moved during the neck bending and the position could have changed during this incident. Especially immediately before extubation, the air was removed from the balloon of the tube so the resistance between the tube and airway mucous membrane was largely reduced, and as the bandage was removed and the RAE tube was about to be extracted, the patient suddenly lifted her upper body and coughed while bending her neck. Hence, it is conjectured that the tube was inserted much deeper than in the experiment where the neck was simply bent, and the end jabbed the mucous membrane of the airway. Macklin [7] has shown the course of air which causes pneumomediastinum in an animal experiment, and also in our case, the air inflow through the damaged mucous membrane of the airway slowly expanded along the bronchovascular sheath to develop pneumomediastinum, and then expanded along the aorta and membrane surrounding the esophagus to develop pneumoperitoneum. In addition, it is considered that it detached the carotid perivascular sheath to develop subcutaneous emphysema in the head and neck, and ruptured into the pleural space causing the pneumothorax. According to the report by Hood and Sloan [8], in patients who suffered injury to the trachea or bronchial tubes, the time taken from damage to diagnosis was diverse, and a delay of 24 hours or more appeared in $66 \%$. In our case, it took about 7 hours from the symptoms to appear from bronchial damage to be diagnosed, and such delay in time is because when there is partial damage to the tracheal wall, the air enters by detaching the adventitia and expanding to form an air sack. This expansion of air can take many hours, and there could be no symptoms until it ruptures into the mediastinum or pleura space [9]. McCoy et al. [10] reported that causes for 
endobronchial intubation were head and neck surgery and laparoscopy which can affect the location of the tube during surgery, incapacity of the anesthesiologist, the movements of the surgeons, and usage of the RAE tube. RAE tubes have been used in $19.5 \%$ of cases where endobronchial intubation occurred. The RAE tube has a relatively large elasticity and structurally forms a sharp angle; thus, it is easy to damage the bronchial tubes when deep intubation occurs, hence there is need for more caution [11]. It is conjectured that also in our case, these structural characteristics of the RAE tube caused more damage to the bronchial tubes than the generally used single lumen endotracheal tube.

In our case, the patient severely vomited several times 5 hours after surgery and the symptoms occurred 2 hours later, and for pneumomediastinum caused by vomiting, there are 2 reasons for esophagus rupture and pulmonary alveoli rupture [12]. Differential diagnosis is performed with fiberoptic esophagoscopy or esophagography, and in our case, an EGD was performed which confirmed that there were no abnormalities in the esophagus. Hence, it is considered that the pulmonary alveoli ruptured from the Valsalva maneuver during vomiting, and the inflow of air expanded through the bronchovascular sheath to cause the pneumomediastinum [7].

Treatment for pneumomediastinum caused by tracheal damage is conservative treatment consisting of bed rest, a supply of oxygen, and use of antibiotics when the symptoms are not severe and the vital signs are satisfactory. When there is severe damage, the vital signs are unstable, and there is a possibility of dyspnea or septicemia, it is better to perform surgical suturing in the early stages [13]. In addition, to reduce pressure, extraction of air through subcutaneous dilacerations and insertion of a subcutaneous catheter are performed; chest tube insertion is performed for a pneumothorax larger than $15-20 \%$ of the thoracic volume, and endotracheal intubation or tracheostomy can be performed when there is severe circulatory disturbance or respiration difficulty $[9,14]$. The patient in our case received conservative treatment consisting of bed rest, oxygen therapy, use of antibiotics, and administration of analgesics together with prompt investigation of the cause after presenting symptoms. The vital signs were not bad so the patient could be observed and monitored, but under the decision that the accompanying pneumothorax and subcutaneous emphysema were severe, chest tube insertion, dilacerations of the subcutaneous emphysema, and aspiration in the retropharyngeal space were performed. Although invasive, it is considered that these prompt decompression procedures prevent cardiac tamponade and an increase in pulmonary vascular pressure caused by air in the vascular sheath and hence, contribute to the faster recovery of the patient. As a method to prevent excessive excitement of the patient while waking up, continuous IV injection of a low concentration of remifentanil has the effect of hemodynamic stability, cough reflex suppression, and pain relief, which can lead to smooth awakening and reduce serious complications as in our case [15]. The patient subsequently received conservative treatment and antitubercular treatment, and recovered enough to be discharged 2 weeks later. Before discharge, we wanted to check what happened to the lesions in the bronchial mucous membrane, but the patient refused so it is a pity that we were unable to follow-up.

From the various cases and literature reviewed above, it is believed that in our case, the struggling and coughing of the patient during regaining consciousness pushed the endotracheal tube into the bronchial tubes, and the end of the tube damaged the bronchial mucous membrane to gradually develop emphysema. The Valsalva maneuver during severe vomiting additionally increased the airway pressure, leaking a large amount of air through the damaged area in a short period of time, which accelerated the emphysema. Pneumomediastinum can be completely treated with conservative treatment, but according to the circumstances, it can proceed to mediastinitis or septicemia and lead to the death for the patient, so diagnosis, treatment, and methods of prevention must be well informed. In addition, the possibility of pneumomediastinum from anesthesia should also be considered and caution should be taken for positive ventilation and endotracheal intubation, and there should be close observation of the patient's state before and after surgery. Especially, there is need for meticulous care after regaining consciousness since the patient's struggling or coughing during extubation can push the endotracheal tube inside to damage the trachea or bronchial tubes, or cause unintended extubation and damage the glottis causing difficulties in maintaining respiration.

\section{References}

1. Jabourian Z, McKenna EL, Feldman M. Spontaneous pneumomediastinum and subcutaneous emphysema. J Otolaryngol 1988; 17: 50-3.

2. Panacek EA, Singer AJ, Sherman BW, Prescott A, Rutherford WF. Spontaneous pneumomediastinum: clinical and natural history. Ann Emerg Med 1992; 21: 1222-7.

3. Zylak CM, Standen JR, Barnes GR, Zylak CJ. Pneumomediastinum revisited. Radiographics 2000; 20: 1043-57.

4. Sohail MA, Kishore K, Stammberger H, Jebeles JA, Luxenberger W. Mediastinal emphysema associated with functional endoscopic sinus surgery. A case report. Rhinology 1995; 33: 111-2.

5. Song CS, Lim SW, Lee SC, Chung SL. Metric study of the upper airway in normal Korean adults with new radiologic lateral view of chest. Korean J Anestheisol 1993; 26: 1016-20.

6. Conrardy PA, Goodman LR, Lainge F, Singer MM. Alteration of endotracheal tube position. Flexion and extension of the neck. Crit 
Care Med 1976; 4: 7-12.

7. Macklin CC. Transport of air along sheaths of pulmonic blood vessels from alveoli to mediastinum: clinical implications. Arch Intern Med (Chic) 1939; 64: 913-26.

8. Hood RM, Sloan HE. Injuries of the trachea and major bronchi. J Thorac Cardiovasc Surg 1959; 38: 458-80.

9. Wagner DL, Gammage GW, Wong ML. Tracheal rupture following the insertion of a disposable double-lumen endotracheal tube. Anesthesiology 1985; 63: 698-700.

10. McCoy EP, Russell WJ, Webb RK. Accidental bronchial intubation An analysis of AIMS incident reports from 1988 to 1994 inclusive. Anaesthesia 1997; 52: 24-31.

11. Black AE, Mackersie AM. Accidental bronchial intubation with RAE tubes. Anaesthesia 1991; 46: 42-3.

12. Schumann R, Polaner DM. Massive subcutaneous emphysema and sudden airway compromise after postoperative vomiting. Anesth Analg 1999; 89: 796-7.

13. Borasio P, Ardissone F, Chiampo G. Post-intubation tracheal rupture. A report on ten cases. Eur J Cardiothorac Surg 1997; 12: 98-100.

14. Herlan DB, Landreneau RJ, Ferson PF. Massive spontaneous subcutaneous emphysema. Acute management with infraclavicular "blow holes". Chest 1992; 102: 503-5.

15. Nho JS, Lee SY, Kang JM, Kim MC, Choi YK, Shin OY, et al. Effects of maintaining a remifentanil infusion on the recovery profiles during emergence from anaesthesia and tracheal extubation. Br J Anaesth 2009; 103: 817-21. 